58 F. Blumenthal, Bericht üb. d. Tätigkeit im Universitätsinstitut f. Krebsforsch.

Figur 4 und $4^{1}$. Längsschnitte durch Mohrrübenscheiben mit Geschwülsten, erzeugt durch Bacillus tumefaciens.

Figur $4^{2}$. Durch Bacillus tumefaciens auf weisser Mohrrübe erzeugte Geschwulstbildung.

Figur 5. Mikroskopisches Präparat des dem Bacillus subtilis ähnlichen, Pflanzentumoren erzengenden Bazillus.

Figur 6. Mohrrübenscheibe mit Tumoren, erzeugt durch Diplococcus tumefaciens.

Figur 7. Mobrübenscheibe mit 'lumoren, erzeugt durch den dem Heubazillus ähnlichen Erreger. (Fig. 5.)

Tafel II.

Figur 1. Sonnenblumenstengel mit Geschwülsten, erzeugt durch Bacillus tumefaciens Smitl.

Figur 2. Zuckerrübe mit Geschwülsten, erzeugt durch Bacillus tumefaciens Smith. Figur 3. Geranie mit Geschwulst, erzengt durch Bacillus tumefaciens Smith.

\title{
4. Die chemisch-biologischen Vorgänge bei der Krebskrankheit.
}

Von Prof. Dr. Ferdinand Blumenthal.

\section{a) Die Ursachen der Krebskachexie.}

Als icl mir bei der Gründung des Instituts im Jahre 1903 ein besonderes, wenn auch kleines chemisches Laboratorium eiurichtete, schwebten mir besonders Arbeiten vor über den Nachweis eines spezifischen Krebsgiftes, dessen Vorhandensein analog dem Diphtherie- oder Tetanustoxin die Existenz eines Krebserregers mehr als wahrscheinlich gemacht hätte. Die Anhänger der parasitären Aetiologie haben, da die Entdeckung des spezifischen Krebserregers ausblieb, vielfach die Beweisführung auf diesem Wege versucht und zwar entweder auf direkte Weise, oder sie haben den indirekten Weg eingeschlagen, indem sie aus der negativen Bilanz des Stickstoff-Stoffwechsels, also aus dem Stickstoffdefizit der Krebskranken gegenüber der Stickstoffaufnahme anf die Existenz eines spezifischen Kachexiegiftes schließen zu können glaubten.

Was den direkten Nachweis der Existeuz eines Krebstoxins anbelangt, so habe ich im Jahre 1903 diese Frage, nachdem sie bis dahin zum Teil bejaht, zum Teil verneint war, mit neuer Technik wieder aufgenommen. Ich habe Glyzerinextrakte, wässerige Extrakte der versehiedenartigsten Krebsgeschwülste Mäusen und Meerschweinchen eingespritzt, ohne daß dieselben bei Mäusen in Dosen bis zu $1 \mathrm{ccm}$, bei Meerschweinchen bis zu 4 ccm eine Giftwirkung zeigten. Auch als ich die Krebsgeschwïlste mit der Hahn-Buchner-Presse analog ihren Hefeversuchen bei einem Druck von 350 Atmosphären auspreßte, zeigte der so gewonnene Saft in 
keiner Weise eine abnorme Giftigkeit für die Versuchstiere. 0,5 bis $1 \mathrm{ccm}$ wurden von $\mathrm{läusen} \mathrm{meist} \mathrm{ohne} \mathrm{Erscheinuug} \mathrm{ertragen.} \mathrm{Das} \mathrm{gleiche}$ war der Fall mit den durch Alkohol gewonnenen Fällungen des Preßsaftes. Auch bei krebskranken Menschen, bei denen solche Einspritzungen behufs aktiver Immunisierung gegen Krebs bis zu 2 ccm Preßsaft pro dosi gemacht wurden, konnte nie ernstere Vergiftungserscheinung nachgewiesen werden. Nur in seltenen Fällen trat leichte Temperaturerhöhung ein, die aber nach kurzer Zeit wieder zurückging. Es endeten alle diese schwierigen und zeitraubenden Versuche nur damit, daß ich ein starkes und in grober Verdünnung wirkendes Krebsgift in den Krebsgeschwülsten nicht nachweisen kounte (1). Damit ist auch die Frage hinfällig, ob eine eventuelle Giftigkeit der Krebsextrakte eine spezifische gewesen wäre. Denn daß unspezifische Gifte in den Organen, insbesondere der Milz von Krebskranken sich anbäufen können, bewiesen Untersuchungen von Fritz Meyer auf der v. Leydenschen Klinik, der die Anbäufung solcher Gifte in der Milz von Krebskranken, insbesondere bei Komatösen hatte nachweisen können.

Friedrich Müller und nach ibm Georg Klemperer hatten gezeigt, dab in den meisten Fällen der krebskranke Organismus beständig an Organeiweiß verliert unter denselben Verhältnissen, die dem gesunden Erhaltung, bzw. Vermehrung des Organeiweißes gestatten, d. h. daß in den meisten Fällen ganz unabhängig von der Nahrungszufuhr ein Zerfall von Gewebseiweil stattindet. Insbesondere gelang es Friedrich Müller nicht bei einem an Peniscarcinom leidenden Kranken, dessen Nahrungsaufnahme nicht behindert war, den Eiweißzerfall durch gesteigerte Nahrungszufuhr auszugleichen. Diese Befunde führten dazu, die Lebre von dem toxogenen Protoplasmazerfall beim Krebs aufzustellen. Denn es schien bierdurch klar zu sein, daß der Krebs eine Krankbeit ist, welche in besonderem Maße schädigend auf deu Stoffwechsel einwirkt. Nun handelt es sich aber darum, ob diese schädigende Einwirkung schon durch bloBes Vorhandensein einer Krebsgeschwulst stattfindet, ohne weitere Komplikationen, oder ob diese erst die Veränderungen im Stoffwechsel hervorrufen, welche dann den großen $\mathrm{N}$-Verlust verursachen. Wir werden daher bei der Betrachtung des N-Stoffwechsels bei Krebskranken völlig unkomplizierte Fälle denen mit Komplikationen entgegensetzen müssen. Ferner müssen wir, da ein Teil der Krebskranken schlecht ernährt werden kann, den Einfluß der Inanition auf den Stoffwechsel beim Gesunden zum Vergleich mit heranzieben.

Unter diesen Gesichtspunkten stellte Braun stein (2)in unserem Institur fest, daß ein kaum noch zu ernährender Kranker mit Krebs der Speiseröhre $3,021 \mathrm{~g}$ Stickstoff und einer mit Carcinoma ventriculi $1,813 \mathrm{~g}$ Stickstoff pro Tag einbüßte. In einem andern Falle von Oesophagus- 
carcinom war der tägliche Stickstoffverlust noch weit böher, $10,65 \mathrm{~g}$, an einem andern Tage sogar 14,757 g. Es war dies ein Tag, an dem der Kranke keine Spur von Nahrung aufnahm. In diesen Fallen hungerten also die Kranken und man kanı hierauf einen erheblichen Teil des Stickstoffdefizits beziehen. Wie groß dieser Anteil ist, läßt sich schätzen, wemn wir die erhaltenen Werte mit denen vergleichen, die jemand, der sonst gesund ist, zeigt, wenn er hungert. Dann können wir erkemmen, inwieweit der Eiweißzerfall eines hungernden Krebskranken sich von dem des gesunden Hungernden unterscheidet. Als gesunder Hungernder diente uns eine hysterische, sonst gesunde Person, welche die Nahrung fast ganz verweigerte; sie schied an drei Tagen, an denen sie keine Nabrung aufnahm, nachdem sie schon vorber wochenlang unteremährt war, folgende Werte aus; $3,65 \mathrm{~g}, 5,1 \mathrm{~g}$, $4,34 \mathrm{~g}$ Stickstoff. Diese Befunde stehen im Einklang mit den Angaben anderer Autoren. v. Noorden fand bei einer Melancholica bei zwei bis fünf Hungertagen $4,8,7,3,6,8,5,8 \mathrm{~g}$ Stickstoff; Senator konstatierte bei ciner weiblichen abstinenten Geisteskranken am vierten Hungertage $6 \mathrm{~g}$ Stickstoff; Friedrich Müller fand bei Hungernden in einem Falle im Ilittel 6,5, im andern Falle $5,3 \mathrm{~g}$ Stickstoff. Die Hungerkünstler Cetti und Succi wiesen zwar in den ersten Hungertagen erheblich höhere Werte auf: Cetti in den ersten vier Hungertagen $12,9 \mathrm{~g}$ im Nittel, Succi $12,8 \mathrm{~g}$; aber abgesehen davon, daB diese Hungerkünstler reichlich Wasser zu sich nalımen, was ein großes Ersparnismittel für den Stickstoffzerfall darstellt, ergaben sich, als die Hungerperiode länger ausgedehnt wnrde, später 4,6 $\mathrm{g}$ und $5,8 \mathrm{~g} \mathrm{~N}$ im Mittel. Es ist also der Wert, den wir bei einer vollkommen hungernden Krebskranken erhielten, $14,75 \mathrm{~g} \mathrm{~N}$, weit höher als die Werte bei solchen Leuten, weiche, ohne eine Krankheit zu haben, keine Nahrung aufnehmen. Daraus könnte man den Schluß machen; daß in diesem Falle der erhöhte Eiweißzerfall auf die Einwirkung der Krebsgeschwulst auf den Gesamtstoffwechsel zu setzen ist. Das ist kaum richtig; denn es handelte sich bei dieser Kranken um ein sehr stark zerfallenes Carcinom, in welchem Bakterien einen erheblichen Liweilzerfall durch Verjauchung hervorbrachten, ferner um eine Pnenmonie der beiden Unterlappen, um eine eitrige Peritonitis und Perikarditis, um Atrophie der Leber und um Blutungen im Magen und Darmkanal. Hier waren also neben der Gesehwulst noch eine Reihe von Organen sekundär erkrankt, und es wird aus diesen Erkrankungen leichter als durch direkte Giftwirkung ron der Geschwulst aus der erhöhte Eiweißzerfall erklärt. Andrerseits zeigte ein andrer Kranker (Oesophaguscarcinom), dessen Tumor aber den Oesophagus nicht so versperte, daß er keine Nahrung zu sich nehmen konnte, sondern der durch Sondierung so weit gebracht war, daß er täglich $7,68 \mathrm{~g}$ Stickstoff im Durchschnitt aufnahm, einen Stickstoffansatz von $1,160 \mathrm{~g}$ Stickstoff pro Tag im Durchschnitt oder $9 \mathrm{~g}$ Eiweiß oder $45 \mathrm{~g}$ Fleisch. 
Dieser Kranke hatte, ehe durch Sondierung seine Stenose erweitert war, 22 Pfund innerhalb 3 Monaten abgenommen, und er setzte nummehr $45 \mathrm{~g}$ Fleisch pro Tag an, als die Nahrungsaufnahme genügend war. Es war also in diesem Falle von einer Krebskachexie nichts zu merken. Es ist ja auch den Chirurgen bekannt, daß, wenn sie bei einem den Pylorus verschließenden Magencarcinom die Gastroenterostomie uachen, die Kranken, die nummelır die gegebene Nahrung wieder ausnützen können, in ungeahnter Weise aufblühen. v. Leyden hat das Verdienst, wie Fr. Müller mit Recht betont, vor allen anderen gezeigt zu haben, was sich durch eine konsequent durchgeführte, rationelle Behandlung, d. h. Ernährung solcher Kranken erreichen läbt. Auch bei anderen Krebskranken sehen wir nicht immer ein N-Defizit. Eine Kranke mit Brustkrebs nahm im Durchschnitt 8 Tage lang 12,96 g Stickstoff mit der Nahrung auf. Sie schied im Harn und Kot zusammen aus 11,0 g Stickstoff im Durchschnitt. Auch diest Kranke setzte eine nicht unbeträchtliche Menge Eiweiß pro Tag an, obwohl sie, wie die Operation zeigte, ein echtes Carcinom in der Brustdrüse batte. Uebrigens beobachtete ähnliches schon Fr. Müller in zwei Fälleo, einem Pankreas- und einem Brustdrüsenkrebs, bei denen in dem ersten Falle nahezu dieselben Werte gefunden wurden, wie bei einer hungernden gesunden Person; und im zweiten Falle war, so lange die Nahrungszufuhr normal war, eine wesentliche Einbuße an Körpereiweiß nicht zu konstatieren. Aus solehen Fälien geht hervor, daß es Krebsfälle gibt, in denen trotz des vorbandenen Krebses eine Kachexie, d. h. ein EiweiBzerfall nicht eintritt.

Die Frage, die wir daber zu erörtern haben, lautet nunmehr: Unter welchen Umständen tritt eine Kachexie beim Krebskranken ein: Die Bedeutung. der Nahrungsaufnahme haben wir schon besprochen. Ein zweites Moment besteht in der Störung der Funktionen solcher für das Leben wichtiger Organe; Tumoren im Nervensystem an Stellen, von denen die regulatoriscbe Tätigkeit anderer Organe abhängjg ist, müssen natürlich zu Störungen in diesen Organen führen. Krebsgeschwülste in der Leber und dem Pankreas können desgleichen die Funktionen dieser Organe beeinflussen. Als drittes Moment kommt in Betracht die Neigung des Krebses zu ulzerieren, und der Eintritt von Infektionserregern in die ulzerierten Stellen; Fieber; Neigung zu Blutungen. Blutverluste erzengen Anämie, und die Infektionen, die ron den ulzerierten Krebsen ausgehen, führen damn zum Bilde der schleichenden Sepsis. Jeder Kliniker kann eine ganze Reihe von Krebsfällen anführen, welche jahrelang "keine Kachexie verursachen, und bei denen sie erst auftritt, wenn Mletastasen in anderen Organen sich einstellen oder ulzerative Prozesse mit sekundärer Infektion sich zeigen. So verhalten sich z. B. zahlreiche Mammacarcinome. Auf 
solche Fälle haben schon R. Virchow und L. Landau aufmerksam gemacht. Aber sie scheinen, je mehr die Diagnostik der Krebse fortschreitet, um so häufiger zu sein. Es zeigt sich nicht selten, daß, wenn solche, lange Zeit in Rube befindlichen - sit venia verbo - Krebsfälle aus irgend einem Grunde alteriert werden, unter Umständen sich gerade dann in verhältnismäßig kurzer Zeit Metastasen bilden und daß, während sie bis dahin verhältnismäBig harmlos erschienen, nunmehr die ganze Bösartigkeit der Krebskrankheit bei ihnen zum Durchbruch kommt.

Wir betracbteten es als unsere Aufgabe, nach den Ursachen zu suchen, weshalb das eine lial die Carcinome so lange obne Kachexie bestehen, und warum in anderen Fällen die Kachexie so frühzeitig vorhanden ist, oder wo sie nicht vorhanden war: plötzlich sich einstellt. Auch v. Hansemann betont, daß es zahlreiche Fälle von Krebs gibt, bei denen durchaus nicht eine ausgesprochene Kachexie besteht, oder bei denen die Kachexie erst im letzten Stadium, kurz vor dem Tode des Patienten auftritt. Hierzu gehören die Krebse des Gesichts, viele Mamma- und Uteruscarcinome. Wenn er aber zu dem SchluB kommt, daß der Krebs als solcher überhaupt keine Kachexie erzeugt, sondern, daß es nur die begleitenden Umstände sind, die bei der Mehrzah! der Fälle die Anämie und Abmagerung hervorbringen, so möchte ich diese Ansicht dabin modifizieren, daß die auch von mir angeführten sekundären Komplikationen doch nur einen Teil der Ursachen der Kachexie darstellen und nichteinmal für alle Fälle zutreffen. v. Hansemann selbst erwähut, daß eine einzige Beobachtung gegen seine Anschauung zu sprechen scheint, uämlich die, daß man auweilen bei Fällen von klinisch perniziöser Anämie ganz kleine Nagenkrebse findet, die sichtlich als solche noch nicht imstande gewesen wären, die Verdauung so weit zu stören, daß durch Vernittlung dieser Störung die Anämie entstanden sei.

Die in solchen Fällen auftretende frühzeitige Krebskachexie ist uns früher ein völliges Rätsel gewesen. Diese kann weder durch Inanition noch durch Ulzeration erklärt werden. Das gilt auch für andere Fälle. In dieses Dunkel haben erst die Fermentforschungen, die im Pathologischen und in unserem Institut zum Teil gemeinsam angestellt wurden, Klarheit gebracht.

\section{b) Die Abartung der eiweißspaltenden Fermente (Enzyme) beim Krebs.}

Petry hatte in der Klinik von Friedrich Kraus in Graz festgestellt, daß die von E. Salkowski entdeckte Autodigestion (Autolyse) besonders stark in dem von ihm untersuchten Krebsgewebe auftrat. Unter Bestätigung dieses Befundes konnte ich gemeinsam mit H. Wolff (3) nachweisen, daß Zusatz von Extrakten von Krebsgewebe (Hammakrebs) zum 
Lebergewebe im Gegensatz zu dem Zusatz anderer Gewebe eine bedeutende Verstärkung der Leberautolyse zur Folge hatte. Wir schlossen darans, daß dies durch ein in dem Krebsgewebe vorhandenes Ferment (Enzym) oder Agens bedingt sei. Die gleiche Tatsache wurde für Krebskuoten in der Leber bei der Einwirkung auf Lungengewebe zur selben Zeit von C. Neuberg in der chemischen Abteilung des Pathologischen Instituts erboben und dabei gezeigt, daß die hierbei entstehenden Produkte eine Umkehr der in der Norm bestehenden Verhältnisse aufwiesen, indem die entstebenden Albumosen nicht wie normal weiter abgebaut wurden. Ferner stellten Neuberg und Milchner fest, daB bei der Autolyse eines Lebercarcinoms Pentosen frei wurden, was sie bei der normalen Leberautolyse nicht beobachteten.

Die Bedeutung der von Neuberg und uns gefundenen Tat sache war für die Biologie des Krebses eine vielfache (4 u. 5).

Erstens zeigte sie uns, daß das autolytische Ferment des Krebsgewebes imstande ist, im Gegensatz zu den autolytischen Fermenten anderer Organe, nicht nur sein eigenes Krebseiweiß, sondern auch das anderer Gewebe abzubauen; zweitens, $d a ß$ dieser Abbau in atypischer Weise verläuft. Diese Tatsachen würden ohne weiteres genügen, um die Erscheinungen der Krebskachexie, soweit es sich dabei um schwere Störungen des N-Stoffwechsels handelt, klar zu machen. Diese Wirkung ist aber nicht konstant. Cm dies zu erklären, müssen wir weiter ausholen. Wir baben gesehen, daßs es Krebsfälle gibt, in denen eine Kacbexie garnicht oder nur sehr spät in die Erscheinung tritt, oder in denen die Kachexie sich zwangslos erklären läßt. Zwangslos, denn es könnte sehr wohl die Wirkung eines Kachexie verursachenden Ferments verdeckt sein durch andere in gleicher Richtung tätige Faktoren, z. B. Blutungen und Ulzerationen, die zu Infektion führen. Für den Fall, daß erst spät die Kachexie auftritt, gilt folgende Betracbtung. Es ist klar, daß das Krebsferment nur dann wirken kann, wenn es in die Zirkulation gelangt. Es ist nun aber bei derben, abgeschlossenen Tumoren wohl kaum anzunehmen, daß hier, da ja die Fermente meist intrazellulär sind und erst bei dem Zerfall der Zelle selbst frei werden, erhebliche Vengen des Ferments in die Zirkulation geraten. Daher ist es durchaus im Einklang mit unserer Auffassung, daß abgeschlossene, nicht zerfallene Carcinome lange Zeit bestehen, ohne stärkeren EiweiBzerfall hervorzurufen. Sobald aber ein Zerfall des Tumors eintritt und durch Freiwerden das Ferment in die Zirkulation gerät, kann es seine eiweißspaltende Wirkung ausüben, mit der auch Störungen in der Art des Eiweikabbaues verbunden sind. Ebenso dürfte sich für kleinere weiche Carcinome, soweit sie an und für sich einen lebhaften Stoffwechsel zeigen, die dabei auftretende frübzeitige Kachexie erklären lassen. 
Um diese von uns dem heterolytischen I) Krebsferment zugeschriebene Bedeutung für die Frage der Krebskachexie erhob sich ein heftiger Streit, insbesondere auch als sein Vorkommen von den Anhängern der parasitären Theorie in ihrem Sinue gedeutet wurde. Von diesen wurde nämlich behauptet, das Krebsferment entspräche in seiner Wirkung dem, was man ron dem gesuchten Produkt des Krebserregers, dem Krebtoxin, erwartet hätte; es sei also etwas Körperfremdes, Ektogenes. Dieser Auffassung ist entgegenzutreten. Das Krebsferment gebört durchaus zu den autolytischen Fermenten, wie sie von E. Salkowski in den normalen Geweben gefunden wurden. Die Fähigkeit, Eiweil anderer Organe abzubauen, welche in Gegensatz zu anderen autolytischen Fermenten nur dasjenige des Krebsgewebes besitzt, kann weit besser durch Abartung des normalen Ferments als durch Absonderung von einem Parasiten erklärt werden.

Nun wurde aber die Tatsache des Vorkommens eines heterolytischen Fermentes bzw. die vermehrte Proteolyse durch Krebsgew ebe iiberhaupt bestrittell. Man behauptete sogar, daB Krebsgewebe die Autolyse andere: Organe hemme usw. Um diese und andere Einwände zu studieren, haben Neuberg und ich gemeinsam mit E. Jacoby (5) eine Anzahl neuer Versuche angestellt, die unsere alten Bef̂nde bestätigen. Ferner hatte ich schon früher festgestellt, daß keineswegs jedes Krebsgewebe die Fähigkeit der vermebrten Proteolyse zeigte. Diese Tatsache spricht nun nicht etwa gegen die Bedeutung der Krebsfermente für die Frage der Krebskachexie, um so weniger, als doch Kachexie nicht in jedem Falle von Krebs vorhanden ist, mithin das Krebsferment auch nicht in jedem Fall, insbesondere in den noch operablen wisksam za sein braucht. Kepinow meinte, daß in den Fällen, in welchen Heterolyse nachgewiesen werden kann, es sich um eine sekundäre Einwanderung von Leukozyten in die Krebsgeschwulst haudelt. Zwar ist bisher nicht festgestellt worden, dab Leukozyten Heterolyse machen können, trotzdem halten wir es für möglich, daß erst sekundär in den Krebsgeschwülsten das beterolytische Ferment sich bildet, da, wie erwähnt, die bei Operation erhaltenen und zur Untersuchung verwandten Krebsgeschwülste häufig keine Heterolyse zeigten, während unsere positiven Ergebnisse mit Leichenmaterial gewonnen wurden, d. h. mit solchen Carcinomen, welche den Tod des betreffenden Individuums herbeigeführt haben. Ob bei der Heterolyse Leukozyten eine Rolle spielen oder nicht, ist schlieBlich für unsere Frage gleichgültig.

Aber es ist auch möglich, daß das heterolytische Ferment zwar von Anfang au in der Krebsgeschwulst vorhanden war, daB aber seine Wir-

1) Autolytische Fermente sind solche, die nur das Eiweiß des Organs abbauen, in dem sie vorkommen; das Krebsferment baut auch anderes Organeiweil. ab, ist daber heterolytisch. 
kung vorerst durch gleichzeitig vorhandene Antifermente oder andere hemmende Körper (Milieuwirkung) beeinträchtigt wurde. Daß Antifermente bei Beginn der Krebskrankheit im Blute und in den Organen nachwęisbar sind und erst im späteren Verlauf der Kraukheit zugrunde gehen, wird nachher genauer beschrieben werden (6).

Daß nun aber tatsächlich eine veränderte und atypische Fermentwirkung auf Eiweißkörper durch die Krebsgeschwülste statthat, geht auch aus den Befunden anderer Forscher hervor. Schon im Jahre 1902 hatte Friedrich v: Müller mit Emerson gezeigt, daß die Verdauung im carcinomatösen Magen weiter geht als im normalen Magen, wodurch das SalzSäuredefizit bei normalem Chlorgebalt seine Erklärung findet: durch die weitergehende Zerlegung des Eiweißes in Polypeptide und eventuell Aminosäuren und Diaminosäuren werden Aminogruppen frei, die ihrerseits Salzsäure binden und dadurch ein Salzsäuredefizit verursachen, während die Gesamtazidität durcb die freigewordenen Karboxylgruppen steigen muß. Friedrich v. Müller meinte, daß diese Beschleunigung der Verdauung im carcinomatösen Mageninhalt einem besonderen autolytischen Ferment zukommt, welches von der ulzerierten oder exkoriierten Oberfläche des Carcinoms in den Mageninhalt gelangt. Dieses Ferment ist nach Fischer imstande, schon so schnell und intensiv Spaltungen vorzunehmen, daß im carcinomatösen Magensaft, im Gegensatz zum normalen, Tyrosin, Leuzin, Arginin und Lysin vorkommen und das Salzsäuredefizit des carcinomatösen Magensaftes als Folge dieses Ferments anzusehen ist. Neubauer und H. Fischer ließen Carcinome und Sarkome auf Glycyltryptophan einwirken und fanden in sehr kurzer Zeit eine äuBerst intensive Spaltung, welche ron gutartigen Geschwülsten, sowie von normalen Geweben erst nach bedeutend längerer Zeit bewirkt wurde. Neben diesen Arbeiten aus der Klinik Friedrichs v. Müller haben die Untersuchungen Emil Abderhaldens und seiner Mitarbeiter dann später große Bedeutung für unsere Frage gewonnen; sie konstatierten, daß aus Krebstumoren (Maus) gewommener Preßsaft bestimmte Polypeptide bzw. Seidenpeptone oft rascher spaltete, als Preßsaft aus Geweben der gleichen Tierart, aus dem der Tumor stammte. Aehnlich verhielten sich die Tumorpreßsäfte gegenüber den Tetrapeptiden. Bei Benutzung von Seidenpepton zeigte sich außerdem ein Abbau durch Preßsäfte der Tumoren, der von dem durch die Preßsäfte normaler Gewebe abwich. Bei Rattentumoren war diese Erscheinung weniger auffallend und konstant als bei Väusetumoren.

Eine Heterolyse der peptolytischen Fermente auf Seidenpepton stellten Abderhalden und L. Pincussobn bei menschlichen Tumoren fest. Besonders deutlich wurden ihre Resultate, wenn sie an Stelle der Peptone ein Tripeptid d-Alanyl-glycyl-glycin nahmen, aus welchem durch den Preßsaft normaler Gewebe durch Spaltung von d-AJauin das Glycyl-glycin ent- 
66 F. Blumenthal, Bericht üb. d. Tätigkeit im Universitätsinstitut f. Krebsforsch.

steht. Durch Preßsaft von Krebsgewebe erfolgte der Abbau so, daB zunächst Glykokoll frei wurde und d-Alanyl-glycin entstand. Der Befund eines typischen Abbaues von Peptonen und Polypeptiden war zwar nicht bei allen Tumoren nachzuweisen, jedoch bei einem bestimmten Tumor in jedem Falle.

\section{c) Die Ausbreitung der fermentativen Abartung auf die übrigen Gewebe.}

Ein Haupteinwand, welcher gegen die Lehre von der von den Krebsgeschwülsten ausgehenden Abartung der Fermentwirkungen erboben wird, besteht darin, daß es sich bei der Feststellung dieser Fermentwirkungen um Reagensversuche d. h. um postmortale Wirkungen handelt. Es war daher von entscheidender Wichtigkeit, festzustellen, ob sich diese Fermentwirkungen von den Geschwülsten aus im Organismus weiter verbreiten. Erst wenn dies gelungen ist, sind wir berechtigt, die Schädigungen, welche wir bei Krebskranken beobachten, auf sie zu beziehen: ferner wäre es möglich, daß die Fermentveränderung auf die Krebsgeschwulst beschränkt bleibt, dagegen nicht in die Zirkulation bzw. in die Organe der Krebskranken übertritt d. h. für den Gesamtstoffwechsel bedeutungslos ist. Zur Eutscheidung dieser Frage wurde das Verhalten der in den Krebsgeschwülsten beobachteten Fermentwirkungen in solchen Organen untersucht, die nicht von einer Metastasenbildung befallen waren; und zwar zuerst, ob aus den Krebstumoren Stoffe in die Zirkulation geraten, welche den autolytischen Zerfall fernliegender Gewebe beschleunigen. Es ergab sich, daß das autolytische Ferment in der noch nicht von einer Metastasenbildung ergriffenen Lunge Krebskranker häufig vermehrt ist (6). Dieser Befund war aber nicht konstant; und wir werden nachher sehen, in wie interessanter Weise sich diese Inkonstanz aufklärte. Nach dieser Feststellung gingen wir dazu über, zu prüfen, inwieweit noch gesunde Teile eines Organs, das mit Krebsknoten durchsetzt war. von der Krebsgeschwulst aus betinflußt wurden. Dabei fand Wolf (14), daß in diesen noch gesunden Teilen einer Krebsleber die Nenge der Albumine doppelt so großwar wie die der Globuline, also Werte aufwies, die von der Zusammensetzung der Leber nicht Krebskranker in dieser Hinsicht erheblich abwichen und sich der Zusammensetzung der Tumoren näherten. Wir schlossen daraus, daß beim Krebskranken die chemische Veränderung der Zellen nicht beschränkt bleibt auf die Krebszellen selbst, sondern auch auf die Organzellen, die noch nicht vom Krebs ergriffen sind, herübergreift. Die Frage wurde dann weiter in unserem Institut und in der chemischen Abteilung des pathrio. gischen Instituts bearbeitet. So konstatierte Yoshimoto (10) eine starke Vermehrung der autolytischen Fermentwirkung in den vom Krebs noch 
nicht befallenen Partien einer mit Krebsknoten durchsetzten Leber und Brahn (12) erhob den gleichen Befund für die Katalasen, Peroxydasen, und fettspaltenden Fermente, d. h. es hatten sich die fermentativen Störungen vom Krebsgewebe in gleichem Sinne auf noch nicht krebsige Teile der Leber fortgesetzt.

Wie schon vorhin ausgeführt, schien es nach unsern ersten Untersuchungen, daB die proteolytischen Fermentwirkungen sich vom Krebsgewebe auch in Organe (Lunge), die nicht von der Tumorbildung befallen waren, fortpflanzen können. Die gleichen Ergebnisse erhielt Brahn bei der Prüfung auf Katalasen und Fettspaltung in der metastasenfreien Leber an Krebs Verstorbener.

Der Befund, daß die noch nicht befalleuen Organe Krebskranker in ihrem fermentativen Verhalten eine sichtbare Beeinflussung durch die Krebsgesebwülste und zwar in der Richtung der in diesen sich zeigenden Störungen aufweisen, ist aber, wie oben erwähnt, nicht konstant. Die Ursachen dieser Inkonstanz hat Brahn (15) neuerdings in interessanter Weise aufgeklärt.

Es üben nämlich nur die Carcinome des Nagens und Darms, des Rektums, des Pankreas und der Gallenblase eineu Einfluß auf die enzymatischen Prozesse in der Leber aus, während Carcinome der Gebärmutter, des Kiefers und der Zunge dies nicht tun. Daraus geht bervor, daß besonders von den im Verdaungstraktus befindlichen Tumoren schwere fermentative Stoffwechselstörungen ausgehen. Diese Befunde stimmen durchaus mit den klinischen Erfabrungen überein, denn die genannten Carcinome des Verdauungstraktus führen meist schon sehr frübzeitig zu dem Bilde der Krebskachexie, während andere Carcinome, falts sie nicht, wie z. B. Zungenearcinome, die Ernährung mechanisch erschweren, verhältnismässig lange in gutem Ernährungszust:tnd sich zu halten pflegen. Einen analogen Befund erhob E. Rosenthal in Budapest bei Krebsmäusen. Bei Bestätigung unserer Ergebnisse ïber das Verbalten der Katalasen bei Krebsmäusen fand er die katalytische Wirkung des Blutes nur herabgesetzt bei Mäusen, denen die Geschwulst im Peritoneum erzeugt war; bei subkutan gesetzten Tumoren dagegen nicht.

Mit ihrem Einfluß auf den Gesamtstoffwechsel ist aber die Bedeutung der Krebsfermente noch keineswegs erschöpft. Sie können auch zur Erklärung für die Metastasenbildung herangezogen werden, indem die Fähigkeit der proteolytischen und peptolytischen Krebsfermente, Eiweiß anderer Gewebe anzugreifen, einen locus minoris resistentiae schaffen kann, wo die Geschwulstzellen haften können. Läßt man diese Betrachtungsweise gelren, so würde sich auch erklären, warum normale Zellen bzw Zellen gutartiger Geschwälste keine Metastasen machen, da ihr autolytisches Ferment nicht wie das der Krebsgeschwulst imstande ist, das Gewebe eines anderen Organs zu verletzen. 
68 F. Blumenthal, Bericht üb. d. Tätigkeit im Universitätsinstitut f. Krebsforsch.

In ähnlicher Weise kann man die Neigung der Krebszellen zum infiltrativen Wachstum sich klar machen. Dieser Gedankengang hatte auch schon Friedrich v. H üller veranlaßt, nach einem proteolytischen Ferment in den Krebszellen zu suchen; jetzt dürfte die Auffiudung der eiweiß. spaltenden Krebsfermente uns den Schlüssel für das Verständnis des destruierenden Wachstums der Krebszellen liefern. Der Einwand, der von $R$ üIf gegen diese Tätigkeit der Krebsfermente erhoben wurde, daß es in der Hauptsache der von den schnell wachsenden Krebszellen ausgehende Druck ist, welcher die deletäre Wirkung auf die Gewebe entfaltet, scheint mir nicht stichbaltig. Denn die Proliferationsfähigkeit bei den gutartigen Geschwülsten stebt häufig in nichts denen der bösartigen Zellen nach, ohne daß es bei ibnen zu einem infiltrativen bzw. destruierenden Wachstum kummt. Mit der Wacbstumsfäbigkeit selbst haben diese Krebsfermente an sich nichts zu tun. Bisher haben wir für die Proliferationsfähigkeit der Krebszellen keine greifbare Unterlage. Man könnte mit Ehrlich an einen besonderen Wucbsstoff denken. Bei pflanzlichen gutartigen Geschwülsten finden wir einen solchen. Wenn z. B. die Gallwespe mit einem Stich in die Pflanze ihren Embryo deponiert, so kann an dieser Stelle eine Geschwulst entstehen, die sogenannte Galle. Tötet man den Embryo ab, so findet gleichfalls die Entwicklung der Galle statt, woraus die Botaniker schlossen, daß es sich um ein Wuchsferment handelt, das mit dem Embryo übertragen wird.

\section{d) Die fermentative (enzymatische) Abwehr des Organismus gegen die Krebszellen.}

Es war oben die Rede davon, daß bei den Versuchen über die Heterolyse der Krebsgewebe Abwehr- oder Antistoffe als Hemmungen für diese in Betracht kommen. Tatsächlich besitzt der Organismus Fermente, die Krebsgewebe stark zu schädigen imstande sind; teils sind diese vorgebildet, teils entwickelt er sie während der Krebskrankbeit.

Die ersten Tatsachen in dieser Richtung wurden in unserem Institut erhoben. So fanden $H$. Wolff und ich, daB das pankreatische Ferment außerordentlich leicht Krebsgewebe verdaut, was nach Bergell, Dörpinghaus und Neuberg und Asher gleichfalls für die aus Krebsgesehwiilsten isolierten Eiweißkörper zutriffr. Dagegen entwickelt sich, wie Brieger und Trebing gezeigt haben, während der Krebskrankheit ein antitryptisches Ferment, das im Blute zirkuỉert und so uns einen Eiublick in den Kampf der Gewebe mit den Krebszellen tun läßt. Dann haben Bergell und Carl Lewin (12) in der gesunden Leber ein Ferment gefunden, welches deujenigen Teil der einfachsten Peptide (Seidenpepton), den das Pankreatin nicht angreift, weiter abbaut, und es wurde ferner nachgewiesen, daß auch das Glycylalanin (ein gegen Pankreatin resistentes Peptid) ge- 
spalten wird. Das Leberferment wirkt nach v. Leyden und Bergell (13) nicht sonderlich auf isoliertes CarcinomeiweiB, auch nicht auf den Tumor im Reagenzglas, aber ganz unvergleichlich stärker und selektiver auf den lebenden Tumor, sei es Carcinom oder Sarkom. Was aber die Bedeutung dieser Befunde erst in das richtige Licht setzt, ist die Tatsache, daß die Leber an Krebs verstorbener Menschen und Tiere diese krebszerstörende Figenschaft vermissen läßt. Sie ist anscheinend bei der Weiterentwicklung der Krebskrankheit verloren gegangen. Ein analoger Befund wurde später im Blut von Freund und Kaminer in Wien und von C. Neuberg im Berliner pathologischen Institut erhoben. Blat nicht Krebskranker löst Krebseiveiß auf, agglutiniert und präzipitiert Krebszellen und Kreb-eiweiB; Blut vorgeschrittener Krebskranker läßt diese Eigenschaft vermissen.

Alle diese Ergebnisse zeigen, daß einerseits die Krebsbildung innerhalb des Organismus präformierte Schutzeinrichtungen (Fermente usw.) zu überwinden hat. Andrerseits hat der Organismus die Fähigkeit, auch während der Entwicklung der Krebsgeschwülste s pezif ische Abwehrstoffe gegen Krebswachstum zu bilden. Der erste Befund dieser Art wurde von Braunstein, einem meiner ersten Mitarbeiter auf dem Krebsgebiete, im Noskauer Krebsinstitut erhoben. Er fand, daß bei Näuseund Rattentumoren die Milz während der Krebsentwicklung die Eigenscluaft gewinnt, das Warhstum der Krebszellen zu hindern; durch Einspritzung von Extrakten derartiger Milzen brachte er die Tumoren bei den behandelten Tieren zur Rückbildung, in einigen Fälen zum Verschwinden. Diese Schutzstoffe in der Hilz sind spezifisch, da sie nur gegen die Tumorart schützen, mit der die Tiere, deren Milz zur Behandlung benutzt wurde, behaftet waren. Die gleichen Schutzstoffe scheinen während der Krebskrankheit aus der $\mathrm{Nilz}$ in die Blutbahn äberzutreten; hierauf dürfte die dann von Abderhalden festgestellte Tatsacbe beruhen, dab das Blut von Krebskranken $A$ bbaufermente für Krebseiweiß enthält. Die Konzentration solcher Abbaufermente ia Blut kann eine so große sein, daß dieses, bei Krebstieren eingespritzt, die Tumoren zur vollständigen Resorption bringt. Neben den spezifischen Antifermenten scheinen sich auch unspezifjsche zu bilden. Hierher dürften die von Brieger und Trebing im Blut Krebskranker gefundenen Antitrypsine zu rechnen sein, die möglicherweise nicht nur dem pankreatischen Ferment, sondern auch der Wirksamkeit des heterolytischen Krebsferments entgegenarbeiten.

Wir sehen aus diesen Tatsachen, daß der Organismus jm Kampf mit den Krebszellen Fermente bildet, welche ibr Wachstum hemmen und sie zu vernichten bestrebt sind. Andrerseits gehen bei diesem Kampfe die in der Leber und im Blute volhandenen präformierten Abwehrkörper (Fermente) zugrunde oder werden neutralisiert (Trypsin). Daß auch 
histologisch ein solcher Kampf der Krebszellen mit den Geweben zu beobachten ist, zeigen die Mitteilungen von Orth, der in den verschiedensten Krebsgeschwülsten neben Stellen des frischesten Wachstums andere nachweisen kounte, in denen unzweifelhaft Heilungsvorgänge vorhanden waren; nur wurden diese Heilungsbestrebungen immer wieder zunichte gemacht durch die Neubildung frischer lebensfähiger Krebszellen. Bei diesem Kampfe braucht es nun keineswegs, wie es nach der klinischen Beobachtung den Anschein haben mag, immer zu einem Unterliegen des Organismus zu kommen. Diejenigen Krebstumoren, welche wir festzustellen in der Lage sind, zeigen vielleicht schon ein verhältnismäßig vorgerücktes Stadium: und ob es nicht öfters dem Organismus gelingt, die allerersten Anfänge der Krebsbildung wieder zum Verschwinden zu bringen, entzieht sich unserer Beurteilung.

\section{e) Schlußfolgerungen.}

Wir haben geseben, daß die zu Krebszellen gewordenen Epithelzellen neue Eigenschaften aufweisen. Diese offenbaren uns ibre Bösartigrkeit, denn sie erklären uns die Erscheinungen ihres infiltrativen Wachstums, der Metastasenbildung, des geschwürigen Zerfalls. Sie führen zu Störungen. deren Gesamtwirkung wir klinisch in der Krebskachexie, d. h. in der Verelendung der Krebskranken erblicken. Außer dem Weg der Fermentprüfung haben wir gesucht, ob wir nicht auch eine Erklärung dieser Bösartigkeit in bestimmten chemischen Veränderungen innerhalb der Krebsgewebe, z. B. im Krebseiweiß nachweisen können. Neben einzelnen vou uns erhobenen interessanten Befunden, die sich aber vorläufig noch nicht zu einem Gesamtbild vereinigen lassen, darf wohl in dieser Richtung als wichtigster gelten die schwere Angreifbarkeit des Krebsgewebes und -eiweißes durch das peptische Ferment des Magens, eine Tatsache, die bei der häufigen Entwicklung von Magenkrebs von besonderem Interesse ist.

Wenn uns also die eben mitgeteilten Forschungsergebnisse der Erklärung der Malignität der bösartigen Zellen näher bringen, so müssen wir uns doch darüber klar sein, daß wir damit erst am Anfange des Einblicks in die feineren chemiscben Vorgänge stehen, welche sich in der Krebsgeschwulst und in den Organen der Krebskranken abspielen. Nur die gröberen Veränderungen sind uns bisher sichtbar geworden. Trotzdem werfen uns diese ein Licht in die Art der Stoffwechselstörungen bei der Krebskrankbeit. Wir können jetzt, auch obne daß wir die Hilfshypotbese der parasitären Entstehung der Krebskrankheit zugrunde legen, das Wesen der Bösartigkeit der Krebszelle und der Krebskacbexie verstehen. Nicht ein bazilläres Krebstoxin, sondern Abartung der normalen Fermentwirkungen eröffnen uns das Verständnis für die Tatsachen der Krebskachexie. Atypische Fermentwirkungen fübren zu einem atypischen Abbau der Eiweiß- 
körper, zu einer Störung in den oxydativen Vorgängen jm Organismus, verändern die normale Blutmischung und verursachen, indem eine Störung die andere bedingt, schlieblich eine Potenzierung der Schädigmngen.

Aus diesen Tatsachen ist zu schließen, daß es sich bei dem Werdegange einer Krebszelle aus einer Epithelzelle um eine chemische A bartung handelt. Das Primäre in der Epithelzelle scheinen die chemischen Únwandlungen zu sein, welche ihr die biologischen Eigenschaften erteilen, die sie zur Krebszelle machen. Damit reiht sicb die Krebskrankheit ein unter die Stofiwechselstörungen, denn man hat eine solche Abartung bestimmter Gruppen in dem Eiweißmolekül bei den verschiedensten Stoffwechselkrankheiten feststellen können. F. Kraus hat das große Verdienst, die Abartung der Eiweißkörper in tierischen Organismus zuerst erörtert und experimentell nachgewiesen zu haben. $\mathrm{Er}$ fand mit Umber beim Diabetes eine Leuzinverarmung, dann stellte ich im Hunger Verarmung der Blutglobuline an ihrer Kohlehydratgruppe fest, und gemeinsam mit Bergell: bei schwerem Diabetes Verarmung der Oxyphenolgruppe des Eiweißes, bei akuter gelber Leberatrophie Schwund der Tyrosingruppe. Mohr und Isaak fanden Verarmung an Diaminosäuren beim Pankreasdiabetes der Hunde, desgleichen Kossel und Wakemann bei der Phosphorrergiftung.

Wenn man nun auch den Krebs als eine Abartung im Stoffwechsel betrachten will, so muls man sich aber auch über gewisse Unterschiede ron den bisherigen bekannten Stoffwechselstörungen klar sein. So verlieren beim Ibiabetes die Zellen die Fähigkeit, Zucker zu verbrennen, es gehen den Zellen Eigenschaften verloren, die sie früıer besaßen. Beim Krebs liegen die Verhältnisse anders. Mag auch die Krebszelle, z. B. des Magens, die Eigenschaft verloren haben, Salzsäure zu sezernieren, so hat sie doch dafür neue Eigenschaften eingetauscht, welche sie früher nicht hatte, nämlich die, das Eiweiß anderer Zellen abzubauen, und zugleich auch die Fähigkeit des unbeschränkten Wachstums. Durch das letztere Phänomen, curch die Metastasenbildung und namentlich dadurch, daß sie, in einen neuen gleichartigen Organismus ïberpflanzt, nicht nur zu haften, sonderı anch zu wuchern und dieselbe Krebskrankheit hervorzubringen imstande ist, unterscheidet sich der Krebs von jeder uns bekannten Stoffiwechselstörung, und er zeigt damit eine Eigenschaft, welche wir bei den Infektionen sehen. Bis zu dem Moment, wo die Krebszelle aus der Epithelzelle fertig gebildet ist, mag es sich um eine zelluläre Abartung handeln, ist aber die Lrebszelle einmal entsianden, so ist durch ihre Existenz ein Keim vorhanden, der den ganzen Organismus vergiftet und dessen Eindringen in einen Organismus gleicher Tierart immer wieder dieselbe Krankheit hervorbringen läßt. Auch losgelöst ron ihrem ursprünglichen Mutterboden vermag sie durch zahlreiche Generationen ihre bösartigen Eigenschaften zu 
bewahren, wie dies durch die Versuche z. B. beim Mäusekrebs immer wieder festgestellt worden ist. DaB die Krebszelle aus einer Organzelle hervorgegangen ist und daß sie als solche keine infektiösen Eigenschaften hatte, kann sicherlich kein Grund sein, der fertigen Krebszelle diese Eigenschaften abzusprechen.

Die geschilderten Ergebnisse der fermentativen Forschungen stellen nur einen Teil jener Vorgärge dar, welche sich beim Krebs innerhalb der Krebszellen und durch diese abspielen. Sie bedürfen der Ergänzung durch die Mikrochemie, deren sich rorzugsweise die histologische Forschung bedient. Diese hat zu Ergebnissen geführt, mit denen die unsrigen in guten Einklang zu bringen sind; ich meine die Anschaungen v. Hansemanns über die Anaplasie, die auf einer vollständigen Aenderung in der physiologischen Beschaffenheit und in der Lebensäußerung der Zellen basiert. Zu gleicher SchluBfolgerung führen die neveren Forschungen Boweris. Dagegen sind sie nicht zu vereinen mit jenen Anschauungen, welche die Krebszelle für eine liegengebliebene, später zur Entwicklung gekommene, dabei nicht wesentlich veränderte embryonale Zelle halten, oder mit jenen, welche die in den Epithelzellen bei der Krebswerdung sich vollziehenden Veränderungen für nebensächlich oder sekundär halten. Danach hätte die Epitbelzelle als Krebszelle gar nicht wesentlich neue Eigenschaften erworben, sondern sie soll nur durch die Schädigung des sie im Verband haltenden Bindegewebes ron jeder Hemmung befreit, die ihr angeborenell Fähigkeiten des unbeschränkten Wachstums usw. entfalten können. Diese Ansicht rergleicht die Krebszelle mit wilden Tieren, welche, solange sie im Käfig eingeschlossen sind, ihre bösartigen Eigenschaften nicht zur Geltung bringen können, wenn der Käfig aber durchbrochen ist, ihre Gefährlicbleit zutage treten lassen. Auf diesem Boden steht ja auch die alte Cohnheimsche Theorie, wonach die Krebszellen versprengte zurückgebliebene embryonale Zellen sind, die aus unbekannter Ursache sich plötzlich auf ihre alte Wachstums- und Fortflanzungstätigkeit besinnen. Die Cohnheimsche Theorie konnte sicb besonders darauf stützen, daß einzeine maligne Tumorarten zweifellos aus embryonalen Zellen sich entwickeln. So richtig dies ist; so hat doch gerade die experimentelle Krebsforschung am Tier gezeigt, dab die Krebszelle nicht einfach eine embryonale Zelle ohne wesentlich neue biologische Eigensehaften sein kann. Demn in zahlreichen Versuchen bei verschiedenen Tumorarten ließ sich feststellen, daß die bösartigen Tumoren von Tier zu Tier gleicher Gattung transplantabel sind, daß sie die unbegrenzte Fähigkeit zur Entwicklung von Krebsgeschwulst und Krankheit in sich tragen, während alle diese Eigenschaften bei den embryonalen Zellen nicht vorhanden sind. Die Tierexperimente der Krebsübertragung beweisen einwandfrei, daß auch die Krebszelle, wenn sie wirklich aus einer embryonalen Zelle entstanden sein sollte, erst eine spezifische Umwandlung 
erfahren haben muB, durch die sie die für die Krebszelle prinzipielle Eigenschaft der malignen Tumorbildung erwirbt.

Als ich im Jahre 1906 zum ersten Male zusammenhängend ïber die chemische Abartung als Ursache für die Nalignität der Krebszelle berichtete, schloß ich meine Al handlung mit folgendem Satze: "In der chemischen Abteilung des Berliner patholngischen Instituts und des mit der 1. medizinischen Klinik verbundenen Krebsinstituts sind diese Vorgänge, wie ich glaube, nicht ohne Erfolg für die Kenntnisse der Biologie des Krebses studiert worden, und wir werden sicherlich noch manche schöne Tatsache auffinden, wenn wir die Ergebnisse der reinen chemischen Forschung und Technik bei der Erforschung der Krebskrankheit anwenden "

In beiden Instituten jst dieses wichtige Gebiet in gemeinsamer Arbeit weiter erschlossen worden, und die Ergebnisse haben die Hoffnungen, die wir hegten, nicht getäuscht. Daß diese Untersuchungen auch für andere Gebiete sich fruchtbar erwiesen haben, hat sich bei den chemotherapentischen Arbeiten gezeigt, auf die wir demnächst zurückkommen werden.

\section{Literaturverzeichnis.}

1. F. Blumenthal, Das spezifische Krebsgift. Festschr. f. E. Salkowski. Berlin. August Hirschwald. 1904.

2. A. Braunstein, Ausscheidung der Chloride usw. Zeitschr. f. Krebsforsch. Bd. 1. H. 3 .

3. F. Blumenthal und H. Wolff, Fermentwirkung bei Krebsgeschwülsten. Med. Klinik. 1905. 22. Jan.

4. F. Blumenthal, Die Malignität der bösartigen Geschwälste. Med. Klinik. 1905. No. 15 .

5. F. Blumenthal, E. Jacoby und C. Neuberg, Zur Frage der autolytischen Vorgänge in Tumoren. Med. Klinik. 1909. No. 42.

6. F. Blumenthal, Die chemischen Vorgänge boi der Krebskrankheit. Wiesbaden. Bergmann. 1906 und 1910. Sep.-Abdr. aus Ascher-Spiro. Bd. 10.

7. F. Blumenthal und B. Brahn, Die Katalasewirkung in normaler und carcinomatöser Leber. Zeitschr. f. Krebsforsch. Bd. 8. S. 436.

8. F. Blumenthal, Die chemische Abartung der Zellen beim Krebs. Zeitschr. f. Krebsforsch. 1907. Bd. 5 .

9. H. Wolff, Zur Kenntnis der melanotischen Pigmente. Hofmeisters Beitr. Bd. 5. H. 10.

10. Yoshimoto, Beitrag zur Chemie der Krebsgeschwülste. Biochem. Zeitschr. Bd. 22. S. 299.

11. B. Brahn, Die Wirkung krebskranker Organe auf den Katalasengehalt der metastasenfreien Leber. Preuß. Akademie d. Wissensch. 1910. Bd. 34.

12. P. Bergell und C. Lewin, Pathogener und spezieller Abbau der Krebsgeschwülste. Zeitschr. f. klin. Med. Bd. 64. 
74 F. Blumenthal, Bericht üb. d. Tätigkeit im Universitätsinstitut f. Krebsforsch.

13. v. Leyden und P. Bergell, Dasselbe. Deutsche med. Wochenschr. 1907. No. 23.

14. H. Wolff, Ein Beitrag zur Chemie des Carcinoms. Zeitschr. f. Krebsforsch. Bd. 3.

15. B. Brahn, Weitere Untersuchungen über Fermente in der Leber von Krebskranken. Sitzungsber. d. Kgl. Preul. Akad. d. Wissensch. 1916. 6. April.

16. F. Blumenthal, Bedeutung der fermentativen Vorgänge beim Krebs. Cancer. 1914. No. 7 .

\section{Innere Behandlung der Krebskranken.}

Von Prof. Dr. Ferdinand Blumenthal.

Im wesentlichen gilt auch heute noch, was ich in meinem zum zebnjährigen Bestehen des deutschen Zentralkomitees für Krebsforschung gehaltenen Vortrage 1910 über die innere Behandlung der Krebskranken gesagt habe, nur dalj einige Gedanken und Forderungen nummehr schärfer als damals ausgedrückt werden können, uachdem sie in der $Z$ wischenzeit vielfache Bestätigung und Ergänzung gefunden haben ${ }^{1}$ ).

Die innere Behandlung des Carcinoms ist auch heute im wesentlichen beschränkt auf die verzweifelten, nicht mehr operablen Fälle. Ũnser Handeln wird immer noch ron dem Satz beherrscht, dak jede bösartige Geschwulst, solange sie operabel ist; auch durch Operation zu entfernen ist. Dann aber bleiben für die innere Therapie fast nur solche Fälle übrig, bei denen nach dem bisherigen Stande der Therapie jeder ärztliche Eingriff als aussichtslos anzusehen ist. Es sind boffinungslose Fälle, welche man dem inneren Kliniker und insbesondere unserem Institut zuweist; und es wäre schon eine große Tat, wenn es den therapeutischen Forschungen gelingen möchte für diese Stadien das tragische Ende wenigstens eine Zeitlang hinauszuschieben.

Aber bisher hat die innere Hedizin auch für den allerersten Beginn der Krebskrankheit kein Heilmittel, das an Wirksamkeit dem Messer des Chirurgen vergleichbar wäre, und so muls es dabei bleiben, daß den Krebsinstituten eine anscheinend aussichtslose Aufgabe zufält. Und so ist es bisher in fast allen Fällen, die wir in Behandlung bekommen, lediglich ein Versuch, den wir machen. Aber die Fülle der hierbei gemachten Beobachtungen and Erfahrungen hat bereits zu Fortschritten geführt, die immer mehr die Berechtigung, ja Notwendigkeit der Krebsinstitute und ihrer Selbständigkeit dartum, in denen die Sorge um die Krebskranken nicht

1) Diese Zeitschr. Bd. 10. H. 1. 